\title{
Androgen Regulates Dimorphic F-Actin Assemblies in the Genital Organogenesis
}

\author{
Liqing Liu a,b Kentaro Suzuki ${ }^{c}$ Eunice Chun ${ }^{d}$ Aki Murashimac, e \\ Yuki Sato $^{f}$ Naomi Nakagatag Toshihiko Fujimori $^{\text {h }}$ Shigenobu Yonemura ${ }^{i}$ \\ Wanzhong $\mathrm{He}^{\mathrm{b}}$ Gen Yamada ${ }^{\mathrm{c}}$

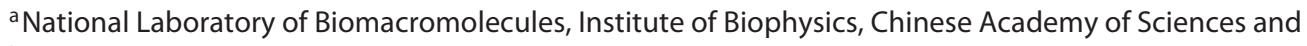 \\ ${ }^{b}$ National Institute of Biological Sciences, Beijing, China; ' ${ }^{c}$ Department of Developmental Genetics, Wakayama

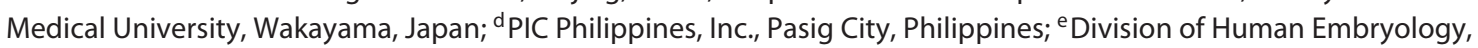 \\ Department of Anatomy, Iwate Medical University, Nishitokuta Yahaba; ${ }^{\mathrm{f}}$ Department of Anatomy and Cell Biology, \\ Kyushu University Graduate School of Medicine, Fukuoka, ${ }^{9}$ Division of Reproductive Engineering, Center for Animal \\ Resources and Development, Kumamoto University, Kumamoto, h Division of Embryology, National Institute for \\ Basic Biology, Okazaki, and 'Department of Cell Biology, Tokushima University Graduate School of Medical Science, \\ Tokushima, Japan
}

\section{Keywords}

Cell migration · Extracellular matrix · F-actin .

Masculinization programming window $\cdot$ Urethral

mesenchymal cells

\begin{abstract}
Impaired androgen activity induces defective sexual differentiation of the male reproductive tract, including hypospadias, an abnormal formation of the penile urethra. Androgen signaling in the urethral mesenchyme cells (UMCs) plays essential roles in driving dimorphic urethral development. However, cellular events for sexual differentiation remain virtually unknown. In this study, histological analyses, fluorescent staining, and transmission electron microscopy (TEM) were performed to reveal the cellular dimorphisms of UMCs. F-actin dynamics and migratory behaviors of UMCs were further analyzed by time-lapse imaging. We observed a prominent accumulation of F-actin with poorly assembled extracellular matrix (ECM) in female UMCs. In contrast, thin fibrils of F-actin co-aligning with the ECM through membrane receptors were identified in male UMCs. Processes for dimorphic F-actin assemblies were temporally identified during an
\end{abstract}

\section{KARGER}

(C) 2017 S. Karger AG, Basel

E-Mail karger@karger.com

www.karger.com/sxd androgen-regulated masculinization programming window and spatially distributed in several embryonic reproductive tissues. Stage-dependent modulation of the F-actin sexual patterns by androgen in UMCs was also demonstrated by time-lapse analysis. Moreover, androgen regulates coordinated migration of UMCs. These results suggest that androgen signaling regulates the assembly of F-actin from cytoplasmic accumulation to membranous fibrils. Such alteration appears to promote the ECM assembly and the mobility of UMCs, contributing to male type genital organogenesis.

(c) 2017 S. Karger AG, Basel

The reproductive organs possess the sexually specific structures to perform the reproductive function. External genitalia are one of the representative organs showing the sexual differences, with the penile urethra being responsible for copulation and urination in the male. The genital tubercle (GT) is the common anlage for the external genitalia of both the male and the female. Androgen regulates masculinization processes of the male reproductive organs contributing to the dimorphic development of the GT. $5 a$-dihydrotestosterone (DHT) is the

Kentaro Suzuki or Gen Yamada

Department of Developmental Genetics, Wakayama Medical University Kimiidera 811-1,Wakayama City

Wakayama 641-8509 (Japan)

E-Mail k-suzuki@wakayama-med.ac.jp or gensan7@wakayama-med.ac.jp 
major androgen for GT masculinization. Testosterone (T) is converted into DHT in the ventral GT mesenchyme by the local $5 \alpha$-reductase [Suzuki et al., 2017]. Mesenchymally-derived androgen signaling is indispensable for the formation of the penile urethra [Miyagawa et al., 2009]. Genetic and molecular mechanisms involved in androgen driven formation of the penile urethra have been studied [Yong et al., 2007; Miyagawa et al., 2009; Chen et al., 2010]. However, the mode of androgen actions regulating cellular differentiation of urethral mesenchymal cells (UMCs) in ventral GT remains to be elucidated.

Androgen actions are required in a specific time window, namely the masculinization programming window (MPW), to regulate multiple processes for the formation of male-type reproductive organs [Welsh et al., 2008, 2014]. Impaired activities of androgen actions within the MPW induce defective male sexual differentiation, such as hypospadias, prostate hypoplasia, and a shorter anogenital distance [Welsh et al., 2008; Suzuki et al., 2015]. Hypospadias is an ectopic opening of the penile urethra in the ventral external genitalia. The incidence of hypospadias ranges from 1:200 1:300 in male newborns [Manson and Carr, 2003; Cunha et al., 2015] and has been reported as increasing over the past few decades due to environmental exposure of anti-androgenic endocrine disruptors [Wilhelm and Koopman, 2006; Cunha et al., 2015]. The critical time window for urethral masculinization is from embryonic day 15.5 (E15.5) to E16.5 in mice [Miyagawa et al., 2009]. Improper exposure of androgen within the time window causes the several degrees of hypospadias. It remains largely unknown how androgen play roles in the differentiation of UMCs within the MPW.

Cytoskeletons regulate cell morphology and cell movement [Alberts, 2008; Heisenberg and Bellaiche, 2013]. The dynamics of actin assembly provide a mechanical basis for cellular behaviors, such as cell mobility and assembly of the extracellular matrix (ECM) [Blanchoin et al., 2014]. Cultured fibroblast cells have been one of the popular models to study cell biology in vitro [Grinnell, 2008; Friedl and Wolf, 2010; Luo et al., 2013]. However, how the assembling pattern of $\mathrm{F}$-actin contributes to the function of mesenchymal fibroblasts during developmental processes remains poorly understood.

In the current study, we observed dynamic sexually different patterns of F-actin in UMCs during urethral formation. Such sexual patterns were regulated by androgen within the MPW. Furthermore, formation of dimorphic F-actin patterns was spatial-temporally consistent with

Androgen-Regulated Dimorphic F-Actin Assemblies the embryonic sexual differentiation processes of several reproductive organs. Of note, dimorphic assemblies of Factin may correlate with the sexual differences of both ECM assemblies and migratory behaviors of UMCs. These results suggest that sexually different assemblies of F-actin may contribute to dimorphic reproductive organogenesis.

\section{Materials and Methods}

\section{Animals}

Mice expressing Actin-Venus (CDB0253K) and Lyn-Venus [Abe et al., 2011] under the control of the Rosa26 Loci were used for time-lapse imaging analysis of tissue slices. $A R^{\text {Flox/Flox }}$ [Sato et al., 2004] females were mated with CAG-Cre [Araki et al., 1997] males to obtain $A R$ knockout males $\left(\mathrm{AR}^{\mathrm{Flox}} / \mathrm{y}\right.$ : $\mathrm{CAG}{ }^{\text {Cre }}$, hereafter designated as ARKO males) [Murashima et al., 2011].

\section{Histology}

Hematoxylin and Eosin (H \& E) and Masson Trichrome staining were performed by standard procedures as previously described [Ogi et al., 2005; Haraguchi et al., 2007]. Tissue sections were prepared into $6 \mu \mathrm{m}$ in thickness for $\mathrm{H} \& \mathrm{E}$ staining and $4 \mu \mathrm{m}$ for Masson's Trichrome staining.

\section{F-Actin and Immuno-Fluorescent Staining for Confocal Microscopy}

GT and other embryonic tissues at various stages were fixed in $1 \%$ PFA at $4^{\circ} \mathrm{C}$ for $1 \mathrm{~h}$ and rinsed and embedded in Cryomold (Tissue-TEK, Sakura, 4566) with OCT compound (Tissue-TEK, Sakura, 4583). Samples were frozen and stored at $-80^{\circ} \mathrm{C}$. Cryosections were dissected into $12 \mu \mathrm{m}$ thickness by a freezing microtome (Leica CM1900), mounted on poly-lysin coated slides, dried briefly for $0.5 \mathrm{~h}$ at room temperature (RT), and were re-fixed with $1 \%$ PFA on ice for $20 \mathrm{~min}$ before staining. F-actin was stained with Alexa-488/647 phalloidin (Invitrogen) following the manufacturer's protocol. In the experiments of F-actin co-staining with other antibodies, phalloidin staining was performed during the secondary antibody incubation. Samples were dissected and fixed with $4 \%$ $\mathrm{PFA}$ at $37^{\circ} \mathrm{C}$ for $\alpha$-tubulin staining. Antibodies for $\alpha$-tubulin (Abcam, Ab52866; 1:300), vimentin (Sigma, V6630; 1:500), fibronectin (Sigma, F1141; 1:500), integrin a5 (BD Pharmingen, 553318; 1:500), and non-muscle myosin IIB (Biolegend, prb-445p; 1:500) were employed.

\section{Chemically Fixed Sample Preparation and Transmission}

Electron Microscopy

Samples were fixed with $2.5 \%$ glutaraldehyde, $2 \%$ formaldehyde in $0.1 \mathrm{M}$ sodium cacodylate buffer ( $\mathrm{pH}$ 7.4) for $2 \mathrm{~h}$ at RT followed by post-fixation with ice-cold $1 \% \mathrm{OsO}_{4}$ in the same buffer for $2 \mathrm{~h}$. After being stained en bloc with $0.5 \%$ uranyl acetate for $2 \mathrm{~h}$ or overnight at RT, samples were dehydrated with ethanol and propylene oxide and embedded in Poly/Bed 812 (Polyscience). Ultra-thin $(70 \mathrm{~nm})$ sections were double-stained with uranyl acetate and Reynold's lead citrate and examined under a JEOL JEM 1010 electron microscope at an accelerating voltage of $100 \mathrm{kV}$.

Sex Dev 2017;11:190-202

DOI: $10.1159 / 000477452$ 
High-Pressure Freezing, Freeze-Substitution, and Transmission Electron Microscopy

Main procedures of sample preparation and transmission electron microscopy (TEM) analysis followed previous descriptions [He et al., 2003] with minor modification to adapt with a different target tissue. The pregnant host animals were anesthetized by isoflorane, and urethral tissues at the proximal GT with thickness $<100 \mu \mathrm{m}$ were dissected quickly from live embryos and mounted flatly on the $100 \mu \mathrm{m}$ depth specimen carriers for high-pressure freezing. Excess space of the carrier chamber was filled with $20 \%$ lipid-rich BSA (ALBUMAXI, Gibco) to remove air bubbles. Specimen were frozen within 310 380 ms under 2,050 bar high-pressure with HPF-compact- 01 (Wohlwend $\mathrm{GmbH}$ ) to obtain a vitrified fixation. The time from embryo dissection to freezing was limited within $40 \mathrm{~s}$ to maintain better physiological conditions. Samples were transferred into screw-typed tubes containing $1 \%$ $\mathrm{OsO}_{4}$ and $0.1 \%$ uranyl acetate in acetone in liquid nitrogen and were subsequently transferred into a Leica EM AFS2 for freezesubstitution. Substitution was programmed as $-90^{\circ} \mathrm{C}$ for $24 \mathrm{~h}$, $-60^{\circ} \mathrm{C}$ for $10 \mathrm{~h}$, and $-30^{\circ} \mathrm{C}$ for $18 \mathrm{~h}$. Temperatures increased slowly during each transition for more than $2 \mathrm{~h}$. Samples were washed 3 times with pure acetone after warming up slowly to $4^{\circ} \mathrm{C}$ and were subsequently warmed up to RT. Urethral tissues were carefully separated from the carrier and infiltrated with Epoxy resin (SPI PON, DDSA, and NMA from SPI-CHEM). Polymerization of resin was achieved by warming at $45^{\circ} \mathrm{C}$ for $18-24 \mathrm{~h}$ and $60^{\circ} \mathrm{C}$ for $48 \mathrm{~h}$. Sections of $70 \mathrm{~nm}$ were placed on 200 mesh fine bar hexagonal grids (Ted Pella, Inc.) coated with a formvar membrane. Positive staining was performed by $3 \%$ uranyl acetate in $70 \%$ methanol on ice for $7 \mathrm{~min}$ and SATO lead in RT for $2 \mathrm{~min}$. Images were recorded with Tecnai G2 Spirit (FEI Corp., Eindhoven, The Netherlands) equipped with $4 \mathrm{k} \times 4 \mathrm{k}$ CCD camera (Gatan Corp.).

Urethral Tissue Slice Culture and Time-Lapse Imaging

GTs with its proximal perineum tissue were dissected at E14.5, E15.5, and E16.5, rinsed, and embedded in 4\% low melting agarose gel (Funakoshi, LM-01) with the distal tip of the GT vertically upward. Agarose gel-fixed tissues were sectioned into slices of $150 \mu \mathrm{m}$ thickness with a Vibratome 7000 (Campden Instrument, Smz). Sections of urethral tissues in the proximal GT were analyzed. Paired urethral tissues were used for comparisons of different treatments. Rat-tail collagen I (BD3542236) was diluted to $2 \%$ and modulated at $\sim$ pH 7.3 with osmotic pressure by $10 \times$ PBS (Wako), charcoal-stripped FBS (Hyclone), $0.05 \mathrm{M} \mathrm{NaOH}$. Urethral tissue slices were rinsed with $2 \%$ collagen gel and mounted on a 4 -well glass bottom dish (Matsunami, D141400). The membrane of the cell culture inserts (MilliCell, PIHT30R48) was prepared into pieces of $9 \mathrm{~mm}$ diameter and rinsed with collagen gel. Urethral tissue slices were covered with the culture insert membrane for a flat mounting to the glass bottom. Collagen gel was fixed at $37^{\circ} \mathrm{C}$ for $10 \mathrm{~min}$. Additional $100 \mu \mathrm{l}$ of $2 \%$ collagen gel was added to the membrane and fixed at $37^{\circ} \mathrm{C}$ for $1 \mathrm{~h}$. A volume of $200 \mu \mathrm{F} 12$ (Gibco) containing $20 \%$ charcoal-stripped FBS was added to each culture well. A physiological concentration of DHT $\left(10^{-8} \mathrm{M}\right)$ was used for the culture. Chemical inhibitors employed in the slice culture system were latrunculin A (Invitrogen) at $5 \mu \mathrm{M}$ and jasplakinolide (Invitrogen) at $5 \mu \mathrm{M}$.

Spinning disk microscopies (Cell Voyager CV-1000, Yokogawa) with $60 \times$ oil lenses were employed for time-lapse imaging experiments. The $10 \mu \mathrm{m}$ sample depth, ranging from 5 to $15 \mu \mathrm{m}$ from the bottom of the tissue slice, was imaged to trace the actin dynamics and cell movement.

\section{Imaging Analysis}

Imaris (Bitplane) was utilized to modulate the brightness and contrast as well as make $10 \mu \mathrm{m} 3 \mathrm{D}$ projections and snapshots at various time points for the time-lapse imaging analysis of Actinvenus indicator mice.

Imaris was also utilized to detect, track, and visualize cell displacement for the time-lapse imaging analysis of Lyn-Venus membrane indicator mice. Cells within $100 \times 100 \mu \mathrm{m}$ scope in the center of each image were tracked, and tracks were manually validated and corrected. Imaris was also applied to obtain quantitative data for the parameter of Track_Speed_Mean, Track_Displacement_ Length, and Track_Length. Such parameters were analyzed in Microsoft Excel to represent the velocity and straightness of the cell movement. Graphpad prism5 was utilized to assemble the cell migratory data.

\section{Results}

\section{Sexually Dimorphic F-Actin Pattern in Genital Development}

Gross morphological sexual difference of the GT becomes initially evident in its ventral side at E16.5 [Suzuki et al., 2002; Yamada et al., 2003a]. The formation of the penile urethra extended into the male GT, whereas it was not formed in the female GT (Fig. 1A, B). Androgen signaling in the mesenchyme around the urethra (hereafter designated as UMCs) is essential for the formation of the penile urethra [Miyagawa et al., 2009; Suzuki et al., 2014]. Intriguingly, cellular morphology of UMCs was significantly different between sexes (Fig. 1C-F). Male UMCs mostly displayed elongated spindle-like cell shape (Fig. 1D, white arrows in Fig. 1F). In contrast, female UMCs showed more rounded morphology (Fig. 1C, white arrows in Fig. 1E).

Cytoskeletons support the dynamic changes of cellular morphology. We thus analyzed the cytoskeletal components of the UMCs. F-actin staining indicates the structural dynamics of actin filaments. Thin F-actin fibrils were observed in male UMCs (Fig. 1I, I', J; online suppl. Video1b; see www.karger.com/doi/10.1159/000477452 for all online suppl. material). On the other hand, a prominently differential pattern of F-actin was observed in female UMCs. F-actin was accumulated into an intensely stained prominent granulation reaching to $2-6 \mu \mathrm{m}$ in diameter. Such accumulation projected thick fibrils out from the central granule (Fig. 1G, G', H; online suppl. Video1a). Moreover, one UMC appeared to possess one such $\mathrm{F}$-actin accumulation based on its number relative to the nuclei number of the UMCs (No. of accumula- 
tions/no. of nuclei $=0.98 \pm 0.09 ; 420$ nuclei were counted, $n=6)$ (data not shown). Vimentin is one of the major components of intermediate filaments in mesenchymal cells, and $\alpha$-tubulin is one of components of the subunit of the microtubule. Contrary to the prominent sexual differences of F-actin, neither the intermediate filaments nor the microtubule showed obvious sexual differences in
UMCs (online suppl. Fig. 1A-H). These results suggest that the sexually dimorphic patterns of F-actin are associated with the dimorphic cellular shapes of UMCs. Next, we investigated sequentially the formation of F-actin assembly from E12.5 to postnatal day 35 (P35). Networks of F-actin fibrils were similarly observed in UMCs of both sexes earlier than E14.0 (Fig. 1K, L; data not shown). Sub-

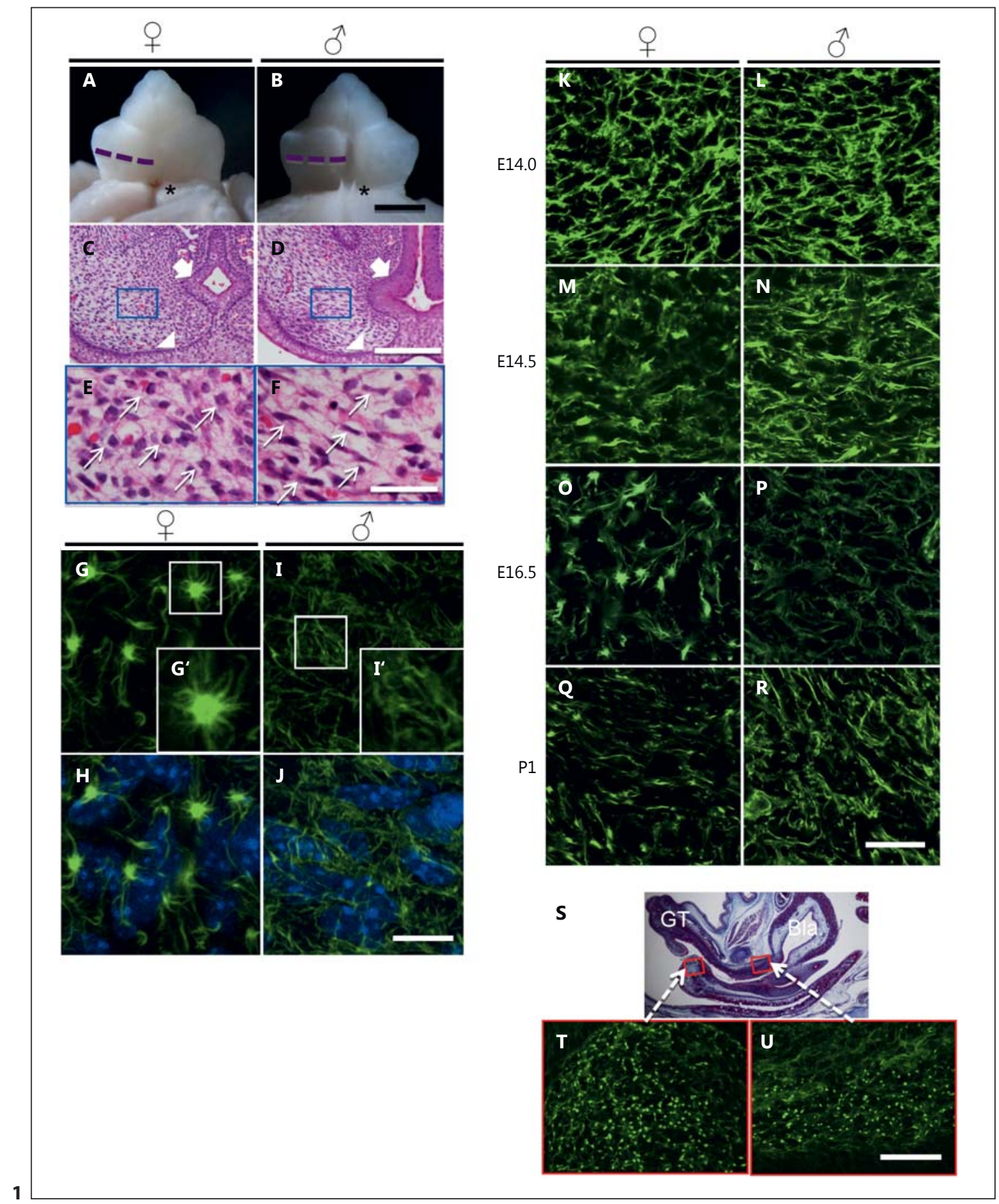

(For legend see next page.)

Androgen-Regulated Dimorphic F-Actin Assemblies
Sex Dev 2017;11:190-202 DOI: $10.1159 / 000477452$ 
sequently, F-actin was assembled into accumulated structures in female UMCs (Fig. 1M) in contrast to the thinner fibrils in male UMCs (Fig. 1N) at E14.5. The most prominent sexual difference of the F-actin assembly was observed between E16.5 and E17.5. Obvious F-actin accumulations were formed in the female UMCs (Fig. 1O). In contrast, much thinner F-actin fibrils developed in the male UMCs (Fig. 1P). Such sexually dimorphic patterns were less prominent at postnatal day 1 (P1) (Fig. 1Q, R). Thereafter, sexual differences of the F-actin pattern become gradually diminished during the postnatal stages (data not shown).

In order to gain insight into the sexual differences of F-actin patterns, we analyzed the F-actin assembly in other tissues of the body trunk and limbs at E16.5. Similar sexual differences could be hardly observed in the skin and the limb mesenchyme (data not shown). However, F-actin accumulations were prominently detected in 2 mesenchymal regions of the female reproductive tract. One location is the lower part of the urethral mesenchyme adjacent to the orifice (Fig. 1S, T), and the other positive region was the upper part of urogenital sinus (UGS) mesenchyme adjacent to the bladder neck region (Fig. 1S, U). This region corresponds to the prostatic budding site in the male urogenital sinus [Hayward et al., 1996] as also suggested by the presence of epithelial budding structures in the corresponding male region at E17.5 (data not shown). On the other hand, no such F-actin accumulation was detected in the urogenital tract of the male. Male mesenchymal cells presented filamentous pattern of F-actin in the corresponding regions (data not shown). Thus, formation of F-actin accumulation appears to be a female-specific feature in several reproductive tissues. We designate herein such accumulation as female-type F-actin accumulation (FTFA).

Fig. 1. F-actin sexual differentiation in genital mesenchymal cells. A, B Gross morphology of the genital tubercle (GT) in female (A) and male (B) mice at E16.5. Asterisks mark the orifice of the urethra in the female (A) and the fused urethra in the male in the proximal GT (B). Scale bar, $500 \mu \mathrm{m}$. C-F H \& E staining of the urethral tissues at the proximal level of the GT indicated by dashed lines in A and B. E, F Urethral mesenchymal cells (UMCs) framed in $\mathbf{C}$ and $\mathbf{D}$. Arrows show the urethral epithelium and arrowheads the ectodermal epithelium (C, D). Female UMCs mostly display rounded cell bodies (C, E, arrows) and male ones display elongated cell bodies (D, F, arrows). Scale bars, $150 \mu \mathrm{m}(\mathbf{C}, \mathbf{D})$ and $50 \mu \mathrm{m}(\mathbf{E}$, F). G, G', I, I' Maximum projection of $6 \mu \mathrm{m}$ thickness confocal images of F-actin stained by phalloidin-Alexa488 in the UMCs. G', I' Enlarged views of the framed area in $\mathbf{G}$ and $\mathbf{I}$. H, J Merged images of F-actin with nuclei. Prominent F-actin accumulation with mul-
Androgen Signaling Modulates Progressive F-Actin Sexual Differentiation in UMCs

Androgen signaling regulates the masculinization of the reproductive tract [Yamada et al., 2003a; Wilhelm and Koopman, 2006; Welsh et al., 2008]. In order to verify the effect of androgen on F-actin assembly, time-lapse imaging systems were developed by using urethral tissue slice cultures. The actin fluorescent indicator mouse line, Actin-Venus [Abe et al., 2011], was employed to trace the actin dynamics of the UMCs at E14.5. Female UMCs developed FTFA-like structures within $12 \mathrm{~h}$ without DHT (Fig. 2B). They gradually achieved a large population of FTFA until 48 h (Fig. 2C-E), whose pattern was similar with the condition at E16.5 (Fig. 1O). The male UMCs developed FTFA without DHT within $36 \mathrm{~h}$ of culture (Fig. 2K-O). In contrast, male UMCs gradually formed thin F-actin fibrils in 2 days culture with DHT (Fig. 2P$\mathrm{T})$. Similar thin F-actin fibrils were also formed in the female UMCs under the treatment of DHT (Fig. 2F-J). Without DHT, F-actin developed into the FTFA regardless of the sex origins of the urethral tissue slices. These results suggest that DHT modulated F-actin patterning into thin fibrils.

To further confirm the involvement of androgen signaling for the formation of the F-actin pattern, we analyzed the GT of androgen receptor (AR) knockout (KO) mice. AR KO males also showed a similar F-actin phenotype like the WT female (online suppl. Fig. 2I, M). Furthermore, administration of DHT could not lead to the formation of the thin F-actin fibrils from FTFAs in AR KO male (online suppl. Fig. 2N, O, P). These results suggest that androgen signaling is essential for the modulation of the F-actin pattern.

DHT modulates the F-actin pattern from the accumulated into the fibrillar form in the female UMCs at E14.5

tiple fibrils projected out from the center in the female UMCs (G, $\left.\mathbf{G}^{\prime}, \mathbf{H}\right)$. Thin F-actin fibrils are formed in the male UMCs $\left(\mathbf{I}, \mathbf{I}^{\prime}, \mathbf{J}\right)$. Scale bar, $10 \mu \mathrm{m}$. K-R Time-course of F-actin sexual differentiation in the UMCs. F-actin patterns in UMCs show no obvious sexual differences between E14.0 female (K) and male (L). There is a partially accumulated pattern in female (M), and bundled fibrillar pattern in male ( $\mathbf{N})$ at E14.5, prominent F-actin accumulations in female (O) and thin fibrils in male (P) at E16.5, and a less accumulated pattern in female (Q) and less thin fibrils in male $(\mathbf{R})$ at P1 compared to E16.5 female and male. Scale bar, $10 \mu \mathrm{m}$. S-U Spatial presence of F-actin accumulation. $\mathbf{S}$ Masson staining of the female urogenital system. Accumulated F-actin staining is present in the lower portion of the urethral mesenchyme under the perineum region $(\mathbf{T})$ and in the upper portion of the urogenital-sinus mesenchyme in the bladder (Bla) neck region (U). Scale bar, $50 \mu \mathrm{m}$.
194

Sex Dev 2017;11:190-202 DOI: $10.1159 / 000477452$
Liu/Suzuki/Chun/Murashima/Sato/

Nakagata/Fujimori/Yonemura/He/Yamada 


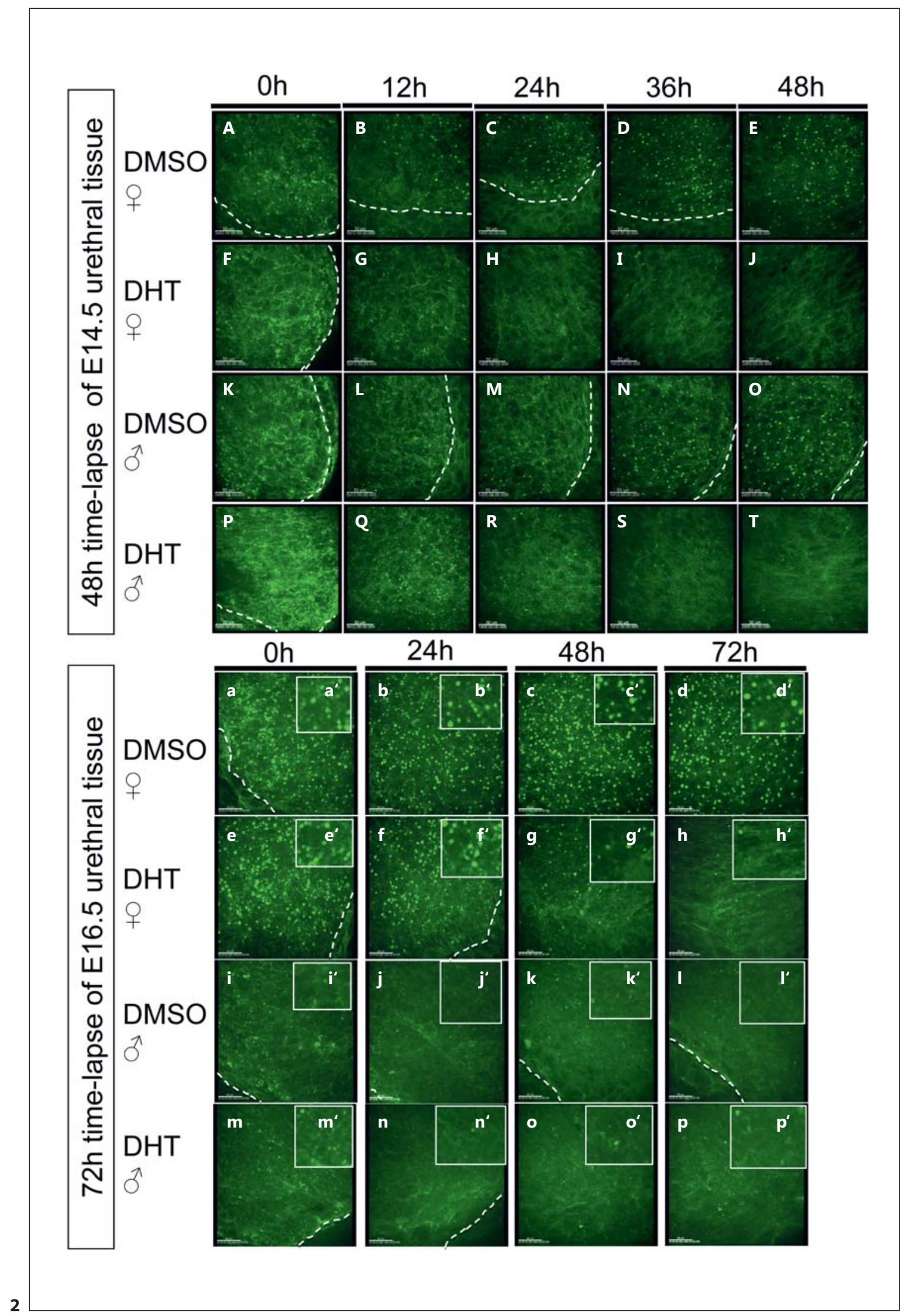

(For legend see next page.) 
within $24 \mathrm{~h}$ (Fig. 2G, H). One day later, namely at E15.5, a longer time was necessary for such DHT-induced modulation of the F-actin pattern in female UMCs (online suppl. Fig. 2F, G, H). These results suggest that the duration of androgen exposure for modulating the F-actin pattern may depend on the developmental stage. To confirm the possibility, we evaluated the timespan to reverse the F-actin sexual pattern of E16.5 specimens. Some FTFAs still remained longer than $48 \mathrm{~h}$ of DHT treatment in female UMCs at E16.5 (Fig. 2g, g', h, h'). In the case of male UMCs, approximately $24 \mathrm{~h}$ were enough to form FTFA-like structures at E14.5 without DHT (Fig. 2M). However, almost no F-actin accumulations were formed even after $72 \mathrm{~h}$ culture of UMCs at E16.5 without DHT (Fig. 2l, l').

\section{Dimorphic Assemblies of the Extracellular Matrix in UMCs}

In order to get insights on the sexually dimorphic pattern of F-actin, we analyzed the subcellular localization of the F-actin structures by TEM. F-actin-like electrondense structures were observed as the mass structure in the cytoplasm of female UMCs (Fig. 3A, area circled by white dashed line in Fig. 3C). Bundle-like structures were observed adjacent to the plasma membrane of the male UMCs (Fig. 3B, arrowheads in Fig. 3D). The physiological status of the cell can be preserved by fast cryo-fixation with high-pressure freezing followed by dehydration through freeze-substitution to reduce extraction of cellular contents [He et al., 2003; McDonald, 2014]. To further characterize dimorphic F-actin assembly, such samples were analyzed by TEM. Electron-dense mass structure was similarly observed in female UMCs (area circled by white dashed lines in Fig. 3E, G). Such FTFA-like structures occupied a large portion of cytoplasmic space, almost devoid of other subcellular organelles inside the FTFA (Fig. 3E, G). Visible F-actin bundles in male UMCs were likely formed adjacent to the plasma membrane (Fig. 3F, H, arrowheads). Such membranous localization of F-actin in the male may promote the physical interaction of the UMC with the ECM in the male.

Fibronectin, one of the key ECM components of mesenchymal fibroblasts, transduces mechanical forces between the intracellular cytoskeleton and the ECM through its membrane receptor, integrin $\alpha 5 \beta 1$ [Pankov and Yamada, 2002]. Fibronectin was assembled into fibrils, which co-aligned with the F-actin fibrils in the male UMCs (Fig. 4C, D, I, J, L; arrows), whereas fibronectin represented a patchy and non-linearized form in female UMCs (Fig. 4A, B, E, F, H). Prominent staining of integrin $\alpha 5$ was detected between the co-aligned fibrils of intracellular F-actin and extracellular fibronectin in male UMCs (Fig. 4K, L; arrows). In contrast, integrin a 5 staining was barely observed between scarcely existing F-actin- and fibronectin-fibrils in the female UMCs (Fig. 4G, $\mathrm{H}$; arrows). These results suggest the presence of more associations of F-actin structures with ECM in the male UMCs.

\section{DHT Regulates Coordinated and Efficient Migration} of UMCs

Dimorphic assemblies of both F-actin and the ECM indicated that there may be sexually different cellular behaviors of UMCs. To investigate this possibility, we analyzed the cellular behaviors of UMCs. Some UMCs migrated from the GT tissue slice into the collagen gel after $48 \mathrm{~h}$ culture of E14.5 specimens. We traced the behaviors of such UMCs derived from Actin-Venus indicator mice. Female UMCs moved randomly and barely contacted each other (online suppl. Video 2a). In contrast, female
Fig. 2. Stage-dependent regulation of F-actin sexual differentiation by androgen through the time-lapse imaging analyses of ActinVenus indicator mice. A-T Imaging snapshots of E14.5 urethral tissue slices of female $(\mathbf{A}-\mathbf{J})$ and male $(\mathbf{K}-\mathbf{T})$ cultured with $(\mathbf{F}-\mathbf{J}$, P-T) or without (A-E, K-O) $10^{-8} \mathrm{M}$ dehydrotestosterone (DHT) in $48 \mathrm{~h}$. Female UMCs at E14.5 develop some F-actin accumulations within $12 \mathrm{~h}(\mathbf{B})$ and gradually achieve a large population of F-actin accumulation within $48 \mathrm{~h}$ (C-E) without DHT (DMSO vehicle control). Female UMCs at E14.5 gradually develop thin F-actin fibrils with DHT (F-J). Male UMCs at E14.5 develop a few F-actin accumulations at $24 \mathrm{~h}(\mathbf{M})$ and achieve a large population of Factin accumulations at $48 \mathrm{~h}$ without DHT (0). Male UMCs gradually develop thin F-actin fibrils with DHT (P-T). a-d Imaging snapshots of E16.5 female (a-h) and male (i-p) urethral tissue slic- es cultured with $(\mathbf{e}-\mathbf{h}, \mathbf{m}-\mathbf{p})$ or without $(\mathbf{a}-\mathbf{d}, \mathbf{i}-\mathbf{I}) 10^{-8} \mathrm{M}$ DHT in 72 h. $\mathbf{a}^{\prime}-\mathbf{p}^{\prime}$ Enlarged views of the central areas of $\mathbf{a}-\mathbf{p}$. Prominent F-actin accumulations of E16.5 female UMCs at 0 h $\left(\mathbf{a}, \mathbf{a}^{\prime}\right)$ were maintained in $72 \mathrm{~h}$ culture without DHT $\left(\mathbf{b}, \mathbf{b}^{\prime}-\mathbf{d}, \mathbf{d}^{\prime}\right)$. Prominent F-actin accumulations of E16.5 female UMCs at $24 \mathrm{~h}\left(\mathbf{e}, \mathbf{e}^{\prime}\right)$ are gradually reformed into thin fibrils $\left(\mathbf{f}, \mathbf{f}^{\prime}-\mathbf{h}, \mathbf{h}^{\prime}\right)$, and a few remnants of F-actin accumulation remain at $72 \mathrm{~h}\left(\mathbf{h}, \mathbf{h}^{\prime}\right)$ in the culture with DHT. Thin F-actin fibrils in E16.5 male UMCs at $0 \mathrm{~h}\left(\mathbf{i}, \mathbf{i}^{\prime}\right)$ were basically maintained in the $72 \mathrm{~h}$ culture without DHT (j, j'$\left.\mathbf{I}, \mathbf{I}^{\prime}\right)$. Thin F-actin fibrils of E16.5 male UMCs $\left(\mathbf{m}, \mathbf{m}^{\prime}\right)$ were maintained in the $72 \mathrm{~h}$ culture with DHT $\left(\mathbf{n}, \mathbf{n}^{\prime}-\mathbf{p}, \mathbf{p}^{\prime}\right)$. All images are Z-stalk projections of $10 \mu \mathrm{m}$ thickness (11 slices, step-height $1 \mu \mathrm{m})$. Dashed lines, epithelial mesenchymal border. Scale bars, $50 \mu \mathrm{m}$.
196

Sex Dev 2017;11:190-202 DOI: $10.1159 / 000477452$
Liu/Suzuki/Chun/Murashima/Sato/

Nakagata/Fujimori/Yonemura/He/Yamada 
UMCs associated and tended to migrate as a group when treated with DHT (online suppl. Video 2b). Male UMCs showed coordinated movements similar with the female UMCs in a DHT-treated condition (online suppl. Video 2d). However, male UMCs without DHT treatment migrated randomly and discretely (online suppl. Video 2c). These results indicate that DHT may promote coordinated cell behaviors of UMCs.
To further investigate the sexual differences of the migratory behaviors of the UMCs, Lyn-Venus membrane indicator mice [Abe et al., 2011] were employed to trace the individual cell movement in urethral tissue slices (Fig. 5; online suppl. Video 3). Male UMCs mostly migrated to the direction of the urethral epithelium (indicated as red line in Fig. 5 A-D), especially with DHT (Fig. 5B, D). However, female UMCs mostly migrated in

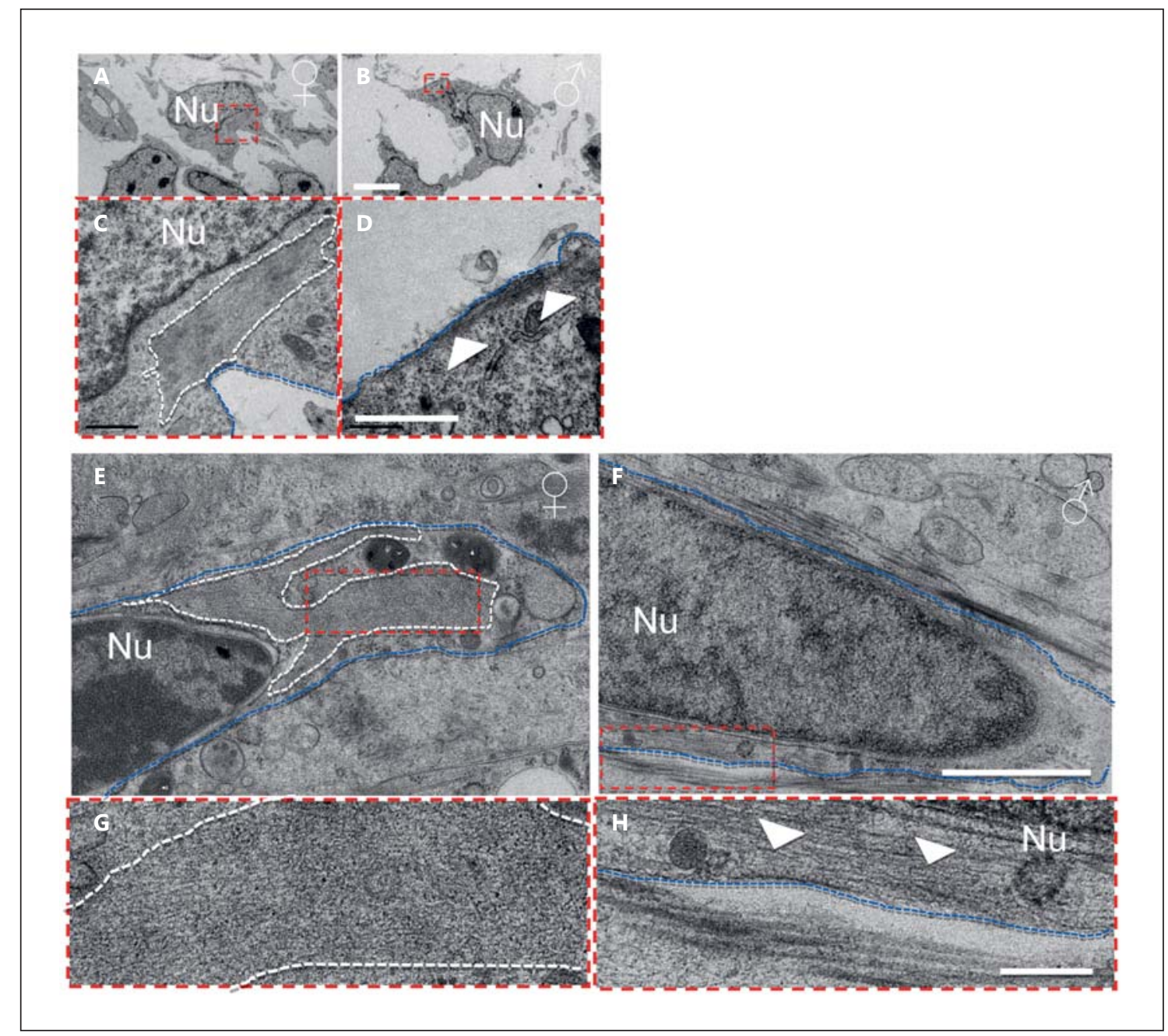

Fig. 3. Dimorphic assemblies of actin filament revealed by transmission electron microscopy (TEM). A-D Chemical-fixed electron microscopy showed the sex-differentiated status of F-actin. An electron-dense mass structure located in the cytoplasm was observed in the female UMCs (C, encircled by a white dashed line). The most prominent bundle-like actin structure was observed underneath the plasma membrane (B, D). The arrowheads point to F-actin structures in male UMCs (D), and blue dashed lines show the plasma membranes. Nu, Nucleus. Scale bars, $5 \mu \mathrm{m}(\mathbf{A}, \mathbf{B})$ and $1 \mu \mathrm{m}$ (C, D). E-H High-pressure freezing, freeze substitution, and electron microscopy showed sex-differentiated status of F-actin in UMCs. The F-actin structure occupied a large portion of the cytoplasmic area (encircled by white dashed lines in $\mathbf{E}$ and enlarged in G), devoid of other cellular organelles inside the FTFA-like structure in the female $\mathrm{UMC}(\mathbf{E}, \mathbf{G})$. F-actin bundles are located adjacent to the plasma-membrane in the male $\operatorname{UMC}(\mathbf{F}, \mathbf{H})$. Arrowheads point to F-actin structures in male UMCs $(\mathbf{H})$, and blue dashed lines show the plasma membranes. $\mathrm{Nu}$, Nucleus. Scale bars, $1 \mu \mathrm{m}$ $(\mathbf{E}, \mathbf{F})$ and $200 \mathrm{~nm}(\mathbf{G}, \mathbf{H})$. 


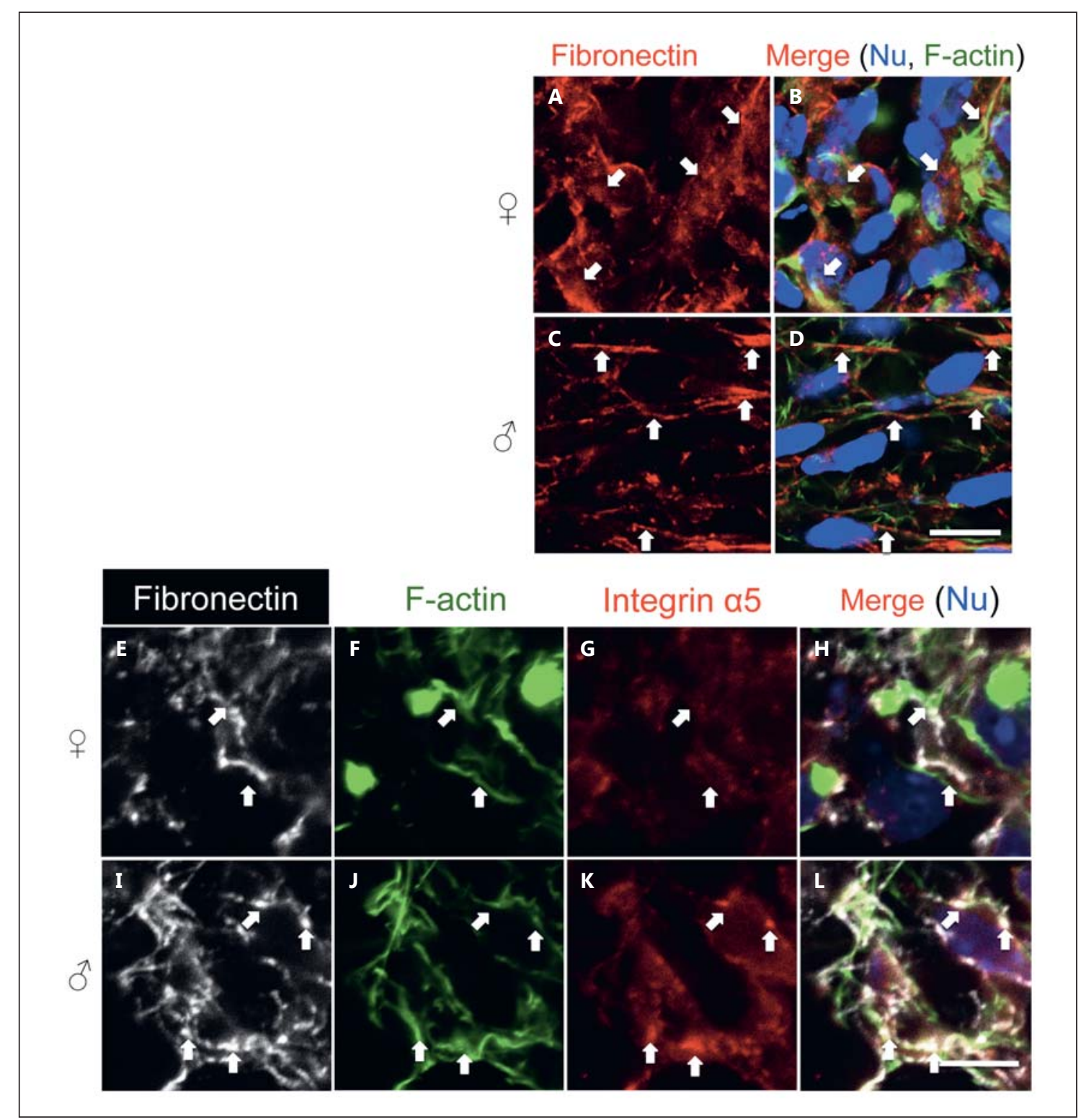

Fig. 4. More prominent co-alignment of fibronectin and F-actin in male UMCs. A-D Expression pattern of fibronectin of E16.5 UMCs. B, D Merged images of fibronectin $(\mathbf{A}, \mathbf{C})$ with $\mathrm{F}$-actin (green) and nucleus (blue). Fibronectin presented a patchy and disorganized form (A), with few thick fibrils colocalized with FTFA in female UMCs (B). Fibronectin was assembled into fibrils (C), which co-aligned with the F-actin fibrils in male UMCs (D). Arrows show selective sites of fibronectin distribution. Scale bar, $10 \mu \mathrm{m}$. Immuno-fluorescent staining of fibronectin (E, I), F-actin
$(\mathbf{F}, \mathbf{J})$, integrin $\alpha 5(\mathbf{G}, \mathbf{K})$, and merged image of 3 fluorescence stainings with nucleus staining $(\mathbf{H}, \mathbf{L})$. Fibronectin of male UMCs (I) present a mostly fibrillar phenotype, co-aligned with intracellular F-actin $(\mathbf{J}, \mathbf{L})$, and there is prominent clustering of integrin $\alpha 5$ between the fibrils of F-actin and fibronectin as indicated by arrows $(\mathbf{K}, \mathbf{L})$. Fibronectin of female UMCs present a patchy form (E), barely colocalized with F-actin structures $(\mathbf{F}, \mathbf{H})$, and there is no prominent integrin $\alpha 5$ staining between the fibrils of fibronectin and F-actin as indicated by arrows $(\mathbf{G}, \mathbf{H})$. Scale bar, $5 \mu \mathrm{m}$. random directions (Fig. 5A). The order of moving velocity of UMCs from low to high was female without DHT, female with DHT, male without DHT, and male with DHT (Fig. 5E). Straightness represents the ratio of dis- placement to the total migrating distance, which indicates the efficiency of the cell movement in one direction. The order of straightness was female without DHT, female with DHT, male without DHT, and male with DHT as the 


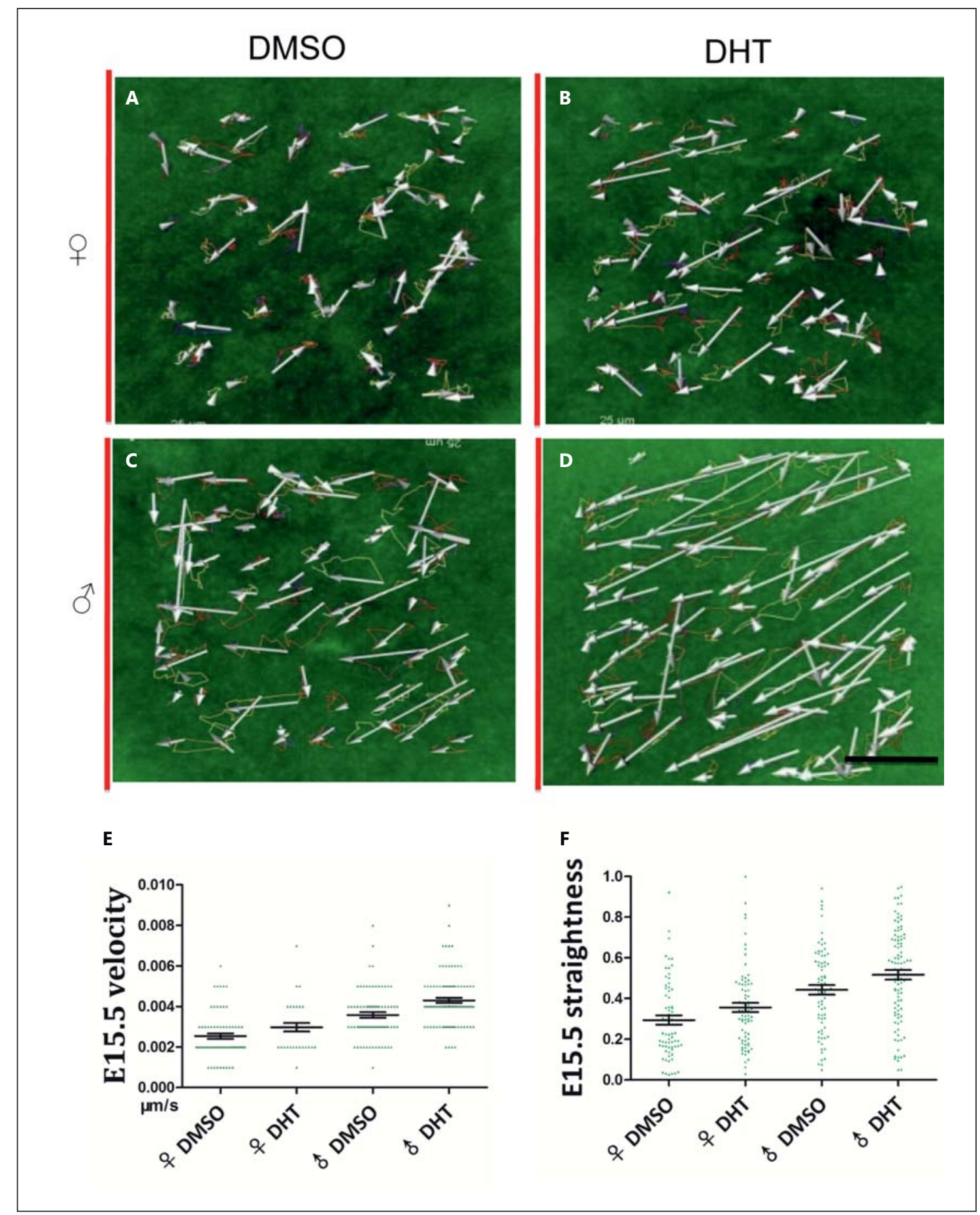

Fig. 5. Androgen regulates more rapid and directional movement of the UMCs. Movement of UMCs in 12-h time-lapse imaging of E15.5 urethral tissue slices from Lyn-Venus membrane indicator mice was traced by Imaris software. Moving route of the UMCs of female $(\mathbf{A}, \mathbf{B})$ or male $(\mathbf{C}, \mathbf{D})$ with $(\mathbf{B}, \mathbf{D})$ or without $(\mathbf{A}, \mathbf{C})$ DHT is displayed by both moving tracks (colored curved lines) and displacement (arrows). Scale bar, $25 \mu \mathrm{m}$. The urethral epithelium is arranged to the left side of urethral tissue slices and is indicated by red lines in A-D. Female UMCs without androgen treatment migrate randomly in all directions (A). Male UMCs with androgen treatment migrate coordinately to the urethral epithelium (D). E Velocity and $\mathbf{F}$ straightness (displacement/length of moving tracks) of UMCs movement are displayed with dispersing point graph. Average velocities of the cells are as follows: female $\mathrm{DMSO}=0.0025 \mu \mathrm{m} / \mathrm{s}$; female DHT $=0.0028 \mu \mathrm{m} / \mathrm{s}$; male DMSO $=$ $0.0036 \mu \mathrm{m} / \mathrm{s}$; male DHT $=0.0043 \mu \mathrm{m} / \mathrm{s}$. Average values of straightness for each group are as follows: female DMSO $=0.29$; female $\mathrm{DHT}=0.36$; male DMSO $=0.44$; male DHT $=0.52$. 
highest (Fig. 5F), which showed a similar tendency with the order of moving velocity. These results indicate that DHT may induce the more efficient and coordinated mobility of the UMCs to the urethral epithelium.

\section{Discussion}

\section{Androgen Regulates the Sexually Dimorphic F-Actin} Pattern

The current results indicate that the F-actin pattern in UMCs shows sexual differences and that androgen signaling is involved in the formation of such pattern. AR function in the mesenchyme is indispensable for fetal sexual differentiation of genital organs in the reproductive tract [Miyagawa et al., 2009]. AR was expressed in the UMCs more prominently in the male between E14.5 to E16.5 [Miyagawa et al., 2009; data not shown]. Secretion of testicular androgen from E13.0 initiates activities of androgen signaling in male embryos [O'Shaughnessy et al., 2006; Dean et al., 2012]. The sexually dimorphic F-actin assemblies in UMCs became initially distinguishable at E14.5, evident at E15.5 and E16.5. The critical time window for the dimorphic development of the GT in mice is reported from E15.5 to E16.5 [Miyagawa et al., 2009]. Sexually dimorphic F-actin patterns were formed around the timing of the MPW. A current study showed that the sexually dimorphic pattern of $\mathrm{F}$-actin was reversible. However, the duration to accomplish sex-reversal patterns of F-actin appeared to depend on the developmental stage. Longer exposure time to modulate androgen signaling was necessary to reverse the female F-actin pattern in late stage (E16.5, more than 3 days) than in earlier stages (E14.5, within 1 days; E15.5, within 2 days). These results suggest that the mesenchymal response to androgen may be different in each stage. Of note, hypospadias-like phenotypes could be induced by anti-androgenic chemicals in the early and mid-stages of the MPW but not in the late MPW [Welsh et al., 2008]. Further analyses are required to understand the correlation between abnormal urethral formation and defects of the F-actin pattern formation.

Sexually dimorphic F-actin patterns were formed not only in the UMCs but also in the mesenchyme of some other embryonic genital regions, including the UGS adjacent to the bladder neck region and the anterior portion of the perineum. These 2 regions also show androgendependent masculinization such as prostate budding and perineum elongation. Furthermore, sexually dimorphic patterns of F-actin in the above reproductive tissues were formed at similar embryonic stages in the MPW. Taken together, the spatio-temporal programs of the formation of sexually dimorphic F-actin patterns may contribute to the sexual differentiation of several reproductive tissues.

\section{Sexually Dimorphic F-Actin Patterns Possibly}

Regulate Distinct Behaviors of UMCs

The F-actin organizations provide a cellular basis for the contractive machineries of the cell to assemble the ECM [Ennomani et al., 2016]. The prominent presence of FTFA may attenuate the membranous distribution of Factin networks, which may decrease the ability of the cell to associate with the ECM. Such a situation might lead to a poor assembly of the ECM in female UMCs.

Fibronectin binds to the cell membrane by its receptor, integrin $\alpha 5 \beta 1$, and regulates the assembly of several other ECM proteins [Pankov and Yamada, 2002; Yamada et al., 2003b]. The expression of integrin $\alpha 5$ between co-aligned F-actin and fibronectin fibrils indicated close associations of fibronectin and F-actin in male UMCs. Such association of F-actin fibrils with fibronectin may transduce mechanical properties from the cell to the ECM facilitating the assembly of the ECM adjacent to the plasma membrane of male UMCs [Yamada et al., 2003b; Alberts, 2008; Leiss et al., 2008]. Well-assembled ECM aligning along cell bodies might promote cellular behaviors of male UMCs in favor of their directional movement.

DHT promoted coordinated cell migration of UMCs in isolated collagen gel environments. Moreover, UMCs migrated more efficiently toward the urethral epithelium of the urethral tissue slices with DHT treatment. Such migration appeared to occur synergistically with a DHT-induced alteration of F-actin patterns. Furthermore, actin polymerization toxins, such as latrunculin $\mathrm{A}$ and jasplakinolide, diminished the F-actin sexual patterns and abolished the mobility of UMCs at the same time (data not shown). Thus, sexually dimorphic patterns of F-actin could play roles in the coordinated directional movement under androgen actions. The formation of the penile urethra involves a dynamic rearrangement of the urethral epithelium, such as the fusion of the urethral plate and the internalization of the urethral tube in the glans. Coordinated migration of UMCs toward the urethral epithelium might positively contribute to such active morphogenetic processes.

Advances in genetic and molecular studies promote the understanding of the mechanism of genital sexual differentiation. Several genes have been identified as essential regulators of male- and female-type reproductive organ formation [Miyagawa et al., 2009; Chen et al., 2010; Chen et al., 2011; Suzuki et al., 2014]. However, the cel-
200

Sex Dev 2017;11:190-202 DOI: $10.1159 / 000477452$
Liu/Suzuki/Chun/Murashima/Sato/ Nakagata/Fujimori/Yonemura/He/Yamada 
lular level of differentiation, particularly mesenchymal characters in the male- and female-type genital organ formation processes remain unelucidated. Dimorphic F-actin assembly in genital organogenesis might be one of the cellular mechanisms for sexual differentiation of reproductive tissues. Further studies are required to reveal the molecular mechanisms of sexually different assemblies of F-actin regulated by androgen.

\section{Acknowledgments}

We thank Drs Benjamin Odermatt, Robert S. Adelstein, Toru Miyazaki, Takashi Seki, Jun Hatakeyama, Nan Tang for their invaluable support, and T. Iba for assistance. We also thank CDB laboratory for Animal Resources and Genetic Engineering for the source of the indicator mice. This work was supported by the Japan
Society for the Promotion of Science KAKENHI Grant Numbers $15 \mathrm{H} 04300,15 \mathrm{~K} 15403$ and $15 \mathrm{~K} 10647$, also by China MOST (973 programs, 2011CB812502, 2014CB849902), and the Beijing Municipal Government.

\section{Statement of Ethics}

All experimental procedures and protocols were approved by the Committee on the Animal Research at the Kumamoto University (B22-198, A23-076), Wakayama Medical University (798), Japan, and by National Institute of Biological Science, Beijing, China (0013).

\section{Disclosure Statement}

The authors have no conflicts of interest to declare.

\section{References}

Abe T, Kiyonari H, Shioi G, Inoue K, Nakao K, et al: Establishment of conditional reporter mouse lines at rosa26 locus for live cell imaging. Genesis 49:579-590 (2011).

Alberts B: Molecular Biology of the Cell, ed 5 (Garland Science, New York 2008).

Araki K, Imaizumi T, Okuyama K, Oike Y, Yamamura K: Efficiency of recombination by cre transient expression in embryonic stem cells: comparison of various promoters. J Biochem 122:977-982 (1997).

Blanchoin L, Boujemaa-Paterski R, Sykes C, Plastino J: Actin dynamics, architecture, and mechanics in cell motility. Physiol Rev 94:235263 (2014).

Chen H, Yong W, Hinds TD Jr, Yang Z, Zhou Y, et al: Fkbp52 regulates androgen receptor transactivation activity and male urethra morphogenesis. J Biol Chem 285:2777627784 (2010).

Chen N, Harisis GN, Farmer P, Buraundi S, Sourial M, et al: Gone with the wnt: the canonical wnt signaling axis is present and androgen dependent in the rodent gubernaculum. J Pediatr Surg 46:2363-2369 (2011).

Cunha GR, Sinclair A, Risbridger G, Hutson J, Baskin LS: Current understanding of hypospadias: relevance of animal models. Nat Rev Urol 12:271-280 (2015).

Dean A, Smith LB, Macpherson S, Sharpe RM: The effect of dihydrotestosterone exposure during or prior to the masculinization programming window on reproductive development in male and female rats. Int J Androl 35: 330-339 (2012).
Ennomani H, Letort G, Guerin C, Martiel JL, Cao $\mathrm{W}$, et al: Architecture and connectivity govern actin network contractility. Curr Biol 26: 616-626 (2016).

Friedl P, Wolf K: Plasticity of cell migration: a multiscale tuning model. J Cell Biol 188:1119 (2010).

Grinnell F: Fibroblast mechanics in three-dimensional collagen matrices. J Bodyw Mov Ther 12:191-193 (2008).

Haraguchi R, Motoyama J, Sasaki H, Satoh Y, Miyagawa $S$, et al: Molecular analysis of coordinated bladder and urogenital organ formation by hedgehog signaling. Development 134:525-533 (2007).

Hayward SW, Baskin LS, Haughney PC, Foster BA, Cunha AR, et al: Stromal development in the ventral prostate, anterior prostate and seminal vesicle of the rat. Acta Anat 155:94103 (1996).

He W, Cowin P, Stokes DL: Untangling desmosomal knots with electron tomography. Science 302:109-113 (2003).

Heisenberg CP, Bellaiche Y: Forces in tissue morphogenesis and patterning. Cell 153:948-962 (2013).

Leiss M, Beckmann K, Giros A, Costell M, Fassler $\mathrm{R}$ : The role of integrin binding sites in fibronectin matrix assembly in vivo. Curr Opin Cell Biol 20:502-507 (2008).

Luo W, Yu CH, Lieu ZZ, Allard J, Mogilner A, et al: Analysis of the local organization and dynamics of cellular actin networks. J Cell Biol 202:1057-1073 (2013).
Manson JM, Carr MC: Molecular epidemiology of hypospadias: review of genetic and environmental risk factors. Birth Defects Res A Clin Mol Teratol 67:825-836 (2003).

McDonald KL: Rapid embedding methods into epoxy and Lr White resins for morphological and immunological analysis of cryofixed biological specimens. Microsc Microanal 20: 152-163 (2014).

Miyagawa S, Satoh Y, Haraguchi R, Suzuki K, Iguchi T, et al: Genetic interactions of the androgen and wnt/beta-catenin pathways for the masculinization of external genitalia. Mol Endocrinol 23:871-880 (2009).

Murashima A, Miyagawa S, Ogino Y, NishidaFukuda H, Araki K, et al: Essential roles of androgen signaling in Wolffian duct stabilization and epididymal cell differentiation. Endocrinology 152:1640-1651 (2011).

Ogi H, Suzuki K, Ogino Y, Kamimura M, Miyado $\mathrm{M}$, et al: Ventral abdominal wall dysmorphogenesis of $m s \times 1 / m s \times 2$ double-mutant mice. Anat Rec A Discov Mol Cell Evol Biol 284: 424-430 (2005).

O’Shaughnessy PJ, Baker PJ, Johnston H: The foetal Leydig cell- differentiation, function and regulation. Int J Androl 29:90-95; discussion 105-108 (2006).

Pankov R, Yamada KM: Fibronectin at a glance. J Cell Sci 115:3861-3863 (2002).

Sato T, Matsumoto T, Kawano H, Watanabe T, Uematsu Y, et al: Brain masculinization requires androgen receptor function. Proc Natl Acad Sci USA 101:1673-1678 (2004). 
Suzuki H, Suzuki K, Yamada G: Systematic analyses of murine masculinization processes based on genital sex differentiation parameters. Dev Growth Differ 57:639-647 (2015).

Suzuki H, Matsushita S, Suzuki K, Yamada G: 5alpha-dihydrotestosterone negatively regulates cell proliferation of the periurethral ventral mesenchyme during urethral tube formation in the murine male genital tubercle. Andrology 5:146-152 (2017).

Suzuki K, Ogino Y, Murakami R, Satoh Y, Bachiller D, Yamada G: Embryonic development of mouse external genitalia: insights into a unique mode of organogenesis. Evol Dev 4: 133-141 (2002).
Suzuki K, Numata T, Suzuki H, Raga DD, Ipulan LA, et al: Sexually dimorphic expression of Mafb regulates masculinization of the embryonic urethral formation. Proc Natl Acad Sci USA 111:16407-16412 (2014)

Welsh M, Saunders PT, Fisken M, Scott HM, Hutchison GR, et al: Identification in rats of a programming window for reproductive tract masculinization, disruption of which leads to hypospadias and cryptorchidism. J Clin Invest 118:1479-1490 (2008).

Welsh M, Suzuki H, Yamada G: The masculinization programming window. Endocr Dev 27: 17-27 (2014).
Wilhelm D, Koopman P: The makings of maleness: towards an integrated view of male sexual development. Nat Rev Genet 7:620-631 (2006).

Yamada G, Satoh Y, Baskin LS, Cunha GR: Cellular and molecular mechanisms of development of the external genitalia. Differentiation 71:445-460 (2003a).

Yamada KM, Pankov R, Cukierman E: Dimensions and dynamics in integrin function. Braz J Med Biol Res 36:959-966 (2003b).

Yong W, Yang Z, Periyasamy S, Chen H, Yucel S, et al: Essential role for co-chaperone Fkbp52 but not Fkbp51 in androgen receptor-mediated signaling and physiology. J Biol Chem 282: 5026-5036 (2007). 